\title{
COMPARISON BETWEEN PCN TUBE AND URETHRAL TUBE URINE SPECIMEN CULTURE IN COMPLICATED UTI
}

\author{
${ }_{1}^{1 \text { st }}$ Year M.Ch, Department of Urology, IGIMS, Patna. \\ ${ }^{2}$ Final Year M.Ch, Department of Urology, IGIMS, Patna. \\ ${ }^{3}$ Senior Resident, Department of Urology, IGIMS, Patna. \\ 4Junior Consultant, MVA, Patna.
}

Kumar Gaurav Mishra1 ${ }^{1}$ Anshul Garg², Pawan Kumar Bharti³, Urvashi Mishra

ABSTRACT

\section{BACKGROUND}

The PCN catheter urine culture is important in cases of complicated urinary tract infections, especially in obstructive uropathy. It is therapeutic as well as diagnostic. The aim of this study was to compare the results of urine cultures obtained either from urethral or percutaneous nephrostomy (PCN) catheters.

\section{MATERIALS AND METHODS}

This study included 78 consecutive patients that underwent PCN at our institution with complicated urinary tract infections (UTIs) between July 2016 and June 2017. Results of urine cultures obtained from the urethral and nephrostomy catheters were compared.

\section{RESULTS}

This study included 56 male and 22 female patients. Mean age of the patients was 15 - 55 years. The main indications were obstructive uropathy due to urolithiasis and PUJO complicated with pyonephrosis 43 (55\%), malignant disease ( $\mathrm{n}=20$; $25 \%$ ), pregnancy $(n=4 ; 5 \%)$ and others $(n=11 ; 15 \%) ; 13$ patients had diabetes mellitus. The most common causative organisms were Escherichia coli, Klebsiella pneumoniae and Pseudomonas aeruginosa. Blood cultures showed the same results for the PCN and bladder urine cultures. The urethral urine culture was positive in 71 patients, while the PCN urine culture in 76 patients.

\section{CONCLUSION}

PCN is an important treatment for the management of pyonephrosis. Cultures from the PCN yield valuable information that is not available from urethral urine cultures and is a guiding tool for antibiotic therapy selection.

\section{KEYWORDS}

Complicated UTI, PCN Tube.

HOW TO CITE THIS ARTICLE: Mishra KG, Garg A, Bharti PK, et al. Comparison between PCN tube and urethral tube urine specimen culture in complicated UTI. J. Evolution Med. Dent. Sci. 2017;6(65):4737-4740, DOI: 10.14260/Jemds/2017/1026

\section{BACKGROUND}

Urinary Tract Infections (UTIs) are problematic conditions frequently encountered by physicians and urologists. A complicated UTI (cUTI) can be associated with any condition that increases the probability of acquiring infection. ${ }^{[1]}$ These UTIs do not typically respond to standard treatment, because of the presence of any anatomical abnormality or pathophysiology. It is generally related to urinary stasis, which provides the time and opportunity for bacteria to adhere to the urothelium and infect the patients. Percutaneous nephrostomy (PCN) is now a well-established technique for providing temporary or permanent drainage of an obstructed urinary system. ${ }^{[2]}$ A contributing factor such as ureteral obstruction may cause renal dysfunction and render the kidneys less effective in concentrating antibiotics in the urine. Ureteral obstruction causes incomplete eradication of bacteria and increases bacterial resistance. [3]

Financial or Other, Competing Interest: None.

Submission 10-07-2017, Peer Review 04-08-2017,

Acceptance 09-08-2017, Published 14-08-2017.

Corresponding Author:

Kumar Gaurav Mishra,

Room No. 31, New MDM,

IGIMS Campus, Rajabazar,

Patna-800014, Bihar.

E-mail: gauravmishra.pmch@gmail.com

DOI: $10.14260 /$ jemds $/ 2017 / 1026$
The most important determinants of complicated UTI caused by resistant strains are previous use of antibiotics and the presence of underlying urological diseases. The microbiological spectrum is wider with a greater prevalence of mixed infections. E. coli remains as the most commonly isolated microorganism $(40 \%-70 \%)$ and other isolated bacteria are Klebsiella, pseudomonas, Staph. aureus, etc. Renal calculi requiring percutaneous nephrolithotomy commonly harbour infectious organisms even when the stone is metabolic in origin. [4] Furthermore, a sterile voided urine does not preclude the presence of pathogens in the urine in pelvicalyceal system ${ }^{[5]}$ or the development of postoperative bacteriuria. ${ }^{[6]}$ Several recent studies have found that the risk of infectious complications after PCNL correlate better with PCN tube urine cultures than preoperative voided urine culture.

The correct and rapid recognition of complicated pyelonephritis is important. When complicating factors are present, antimicrobial resistance is more common and the response to therapy is often disappointing even with agents active against the pathogen. The aim of this study was to evaluate UTI and compare urine culture results obtained from PCN tube and urethral catheter. ${ }^{[7]}$

\section{MATERIALS AND METHODS}

After obtaining informed consent from each participant, 78 consecutive patients with complicated UTI who underwent PCN procedure at our institution between July 2016 and June 
2017 were examined. Results of urine cultures obtained from urethral and nephrostomy catheters were compared. We defined a positive culture as a clean-catch midstream urine specimen with a growth of $105 \mathrm{cfu} / \mathrm{mL}$ of a single microorganism or mixed flora with predominant species. ${ }^{[8]}$ Negative urine culture was defined as no growth, insufficient growth or a mixed microbial flora with no predominant organism. Patients with positive preoperative urine cultures were treated with suitable antibiotics based on the susceptibility test results. For patients with mixed flora but a predominant organism on urine culture, a specific antibiotic effective on this organism was selected. If culture results contained mixed organisms, contamination was established and these results were excluded from the analysis so this is the exclusion criteria of our study.

A drainage procedure was considered when clinical improvement has not been noted within $48-72 \mathrm{~h}$ of adequate antimicrobial treatment. If concomitant obstructive uropathy still existed, the upper urinary tract was decompressed with PCN.[9]

In this study, all cases of PCN were performed under ultrasonographic guidance under local anaesthesia with the patient in a prone or prone-oblique position. Prophylactic antibiotics were routinely administered and routine investigations including complete blood count, viral markers, renal function tests, coagulation profiles and radio imaging studies were obtained before the PCN. After the initial PCN, an antegrade nephrostogram was performed between 48 and 72 hours to delineate the exact nature of the obstruction and configuration of the urinary tract. All medical records were reviewed for aetiology, clinical presentation, laboratory and microbiological culture reports, definitive treatment, complications and patient outcomes.[10] In situations where a culture grew multiple organisms with only one being a urease producing organism, that case was grouped in the category of urea-splitting organisms. When a culture grew multiple organisms without urease producing organism or had more than one urease producing organism, that case was grouped in multiple organisms. Multiple organisms were cultured in $6 \%$ of preoperative urine cultures and $8.3 \%$ of PCN culture. The identity of organisms cultured was missing for one patient in each of the preoperative urine cultures and PCN culture groups.

\section{Statistical Analysis}

For statistical analysis, the Statistical Package for the Social Sciences (SPSS Inc., Chicago, IL, USA) software package was used. $\mathrm{P}<0.05$ was accepted as the cut-off value for the level of statistical significance. The Pearson chi square method was used to compare parameters between the two groups.

\section{RESULTS}

This study population consisted of 56 male and 22 female patients. The mean age of the patients was $15-55$ years. The main indications were obstructive uropathy due to pyonephrosis with urolithiasis and PUJO 43 (55\%), malignant disease 20 (25\%), pregnancy $4(5 \%)$ and others $11(15 \%)$. PCN was performed on 44 right sides and 34 left sides of the kidneys. All procedures were performed under ultrasound guidance in prone and oblique prone position under local anaesthesia.
During all procedures, major complications were sepsis $(n=4)$ and haemorrhage $(n=2)$ requiring transfusion. In all these patients, the bleeding ceased after prolonged tube drainage within one week. Minor complications within 30 days of follow-up were recorded in 18 patients. Retroperitoneal urine extravasation was encountered in 1 of 78 patients and treated conservatively. Tube-related complications, such as catheter dislodgement and leakage from the catheter occurred in 11 patients.

Causative organisms were Escherichia coli, Klebsiella pneumonia, Pseudomonas aeruginosa, Staph. aureus, Enterococcus spp., Candida albicans, Staphylococcus epidermidis and Acinetobacter spp.

Blood and PCN culture results were not significantly different. Culture results of urine specimens obtained from urethral catheter and PCN tubes differed in 5 patients. Urethral urine culture was positive in 71 (91\%) patients, while the PCN urine culture in 76 (97\%) patients without any statistically significant intergroup differences $(p=0.09)$. However, three patients had positive PCN, but negative urethral urine culture results. Negative PCN $(n=2)$ and urethral $(n=7)$ urine culture results were detected in respective number of patients.

E. coli and Klebsiella spp. were commonly sensitive to amikacin (85\% - 90\%), ceftriaxone $(20 \%-25 \%)$, Levofloxacin (50\% - 55\%), imipenem-cilastatin (100\%), nitrofurantoin $(65 \%-70 \%)$ and ampicillin $(10 \%-15 \%)$ in urethral urine cultures, respectively. These microorganisms detected on PCN urine culture media were sensitive to amikacin (86\% - 90\%), ceftriaxone $(24 \%-25 \%)$, levofloxacin (50\% - 55\%), imipenem-cilastatin (100\%), nitrofurantoin (65\% - 70\%) and ampicillin (12\% - 15\%).

\section{DISCUSSION}

The treatment of complicated UTIs in the presence of urinary tract obstruction requires effective antibiotic therapy as well as appropriate urological intervention to prevent septicaemia and recurrent UTIs.[11] Patients who have complicated UTIs should be hospitalised and empirical treatment may include intravenous ampicillin and amikacin or alternatives such as ciprofloxacin, levofloxacin, ceftriaxone, aztreonam and imipenem-cilastatin.[12] The choice of empirical antibiotic treatment should be based on local antibiotic therapy protocols. Empirical therapy of complicated UTIs should usually include an intravenous antipseudomonal agent. Targeted therapy should be initiated once susceptibility data are known. Agents commonly prescribed include aminoglycosides, beta lactamase inhibitor combinations, imipenem, third generation cephalosporins and fluoroquinolones. Indiscriminate use of quinolones and cephalosporins is strongly discouraged because of increasing bacterial resistance. Therapy is usually switched from parenteral to oral as soon as possible. According to literature, analysis of bladder urine may not be the same as renal urine culture in obstructive kidneys. Sancaktutar et al[13] showed that bladder urine culture was positive in 13 (39.4\%) of 33 patients; however, percutaneous nephrostomy urine culture was positive in most of the patients ( $n=25,78.1 \%)$. Lei et al should have done a separate renal urine culture, which could be obtained from percutaneous access needle at the beginning of the procedure. We believe that these missing data could be added to the literature. 
The negative urethral urine susceptibility test results did not correlate well with the upper urinary tract infection. It has been reported that urine culture and positive calculus culture were better predictors for potential urosepsis than urethral urine specimens. Studies also have shown that the disparity between urethral urine and PCN tube urine cultures ranged from $37 \%$ to $52 \%$. The reason for the poor correlation between PCN and urethral urine culture results could be related to complete ureteral obstruction, which often prevents microorganisms from travelling from upper urinary tract down to the bladder.[14] In this study three patients had positive PCN, but negative urethral urine cultures. In the setting of upper urinary tract collecting system, obstruction was complicated by infection. However, drainage is an emergency and in many such cases percutaneous rather than retrograde drainage may be best unless retrograde drainage can be obtained expeditiously and assuredly. Percutaneous nephrostomy tubes and retrograde ureteral stents are generally equivalent in their capacity to resolve fever in patients with upper urinary tract obstruction, but given the patient's circumstances may dictate a preference for one access instead of the other. ${ }^{[15]}$

In our patients with positive urine cultures E. coli was the most common organism, which accounted for $60.0 \%$ of the positive cultures. Escherichia coli is the most frequent aetiological agent in any UTI, whereas other gram-negative bacteria such as Klebsiella spp. or Proteus spp. should be considered as well. A recent study that included 800 patients with complicated UTI, most of which concerned with complicated pyonephrosis found that Escherichia coli and Klebsiella spp. were responsible for $63.0 \%$ and $7.0 \%$ of the infectious episodes, respectively. In our study, ESBLproducing microorganisms were detected in 58\% - 60\% of our patients, which was consistent with previous reports. This aetiological spectrum and the ever-increasing incidence of ESBL-producing multi-resistant microorganisms demand a special effort in the aetiological diagnosis of complicated pyonephrosis.

Percutaneous nephrostomy and ureteral stenting for the treatment of pyonephrosis were compared and it was reported that both percutaneous and retrograde routes were effective. However, $62.0 \%$ of PCN urine cultures were positive compared with only $19.0 \%$ of retrograde catheter urine cultures. This is because retrograde ureteral stenting has a number of disadvantages in the management of pyonephrosis compared with PCN. Ureteral stent has few disadvantages as it usually comes in smaller sizes, which provides less effective drainage and often needs to be performed in the operating room under general anaesthesia. Furthermore, there is the risk of perforating the ureter during manipulation. In addition, bacteraemia and septicaemia may flare up under the pressure of the irrigation fluid.[16]

In addition, it is clear that the microbiology of PCN urine culture has changed dramatically during the last generation from predominantly gram-negative to now predominantly gram-positive organisms.[17]

There are a number of possible explanations. Several groups have documented the decreasing frequency of struvite stones in the adult and paediatric/ neurogenic patient population. In this series, proteus and Morganella species were identified in only $13.7 \%$ (41 of 300 ) of positive PCN urine cultures and $17.2 \%$ ( 61 of 354 ) of positive preoperative bladder urine cultures.[18]

In most patients, the collecting system is localised by either fluoroscopy or ultrasound guidance. When the collecting system is moderately or severely dilated, ultrasound guidance is successful in aiding entry into the collecting system in $85 \%$ to $95 \%$ of patients. Conversely, in only mildly dilated collecting system the success rate may be as low as $50 \%$.

Regardless of the degree of renal collecting system dilatation if renal function is poor (the usual case with obstruction) or if contrast cannot be administered, ultrasound guidance, preferably real time is the preferred way to localise the collecting system. Once the collecting system has been punctured, the remainder of the procedure is usually performed using fluoroscopic guidance alone, but in our case all PCN procedures have been done under ultrasound guidance. Numerous techniques have been described for PCN placement, all of which entail imaging and puncturing the collecting system, dilating the tract and then placing a catheter. Planning the puncture site is crucial step in the procedure. The skin puncture site is chosen, so that the catheter enters the flank in the posterior axillary line and courses subcostally below the inferior margin of the $12^{\text {th }}$ rib. This is done to minimise the chance of injury to the intercostal artery located on the inferior margin of the rib and also to decrease the chance of intercostals nerve irritation which can be very painful. If the intercostals space between the $11^{\text {th }}$ and $12^{\text {th }}$ ribs must be used for the procedure, care should be taken to stay close to the superior aspect of the $12^{\text {th }}$ rib to avoid neurovascular bundle. The puncture and therefore the nephrostomy tract should traverse the renal parenchyma before entering the collecting system, so that the parenchyma can provide a secure seal around the catheter. Entry into the collecting system should ideally be into a posteriorly directed calyx through the relatively avascular posterolateral plane of kidney, the so called avascular plane/ line of brodel. This is the zone where the renal artery divides into its major ventral and dorsal branches and lies at the junction of the anterior two-thirds and posterior one-third of the kidney, just posterior to the to the lateral convex border of the kidney. The posterior calyces point to this plane and can be identified as the more medially positioned calyces. Entry into the posterior calyces minimises the risk of entering the large vessels in the renal hilum. If PCN is being performed for drainage alone, virtually any posterior calyx can be suitable for access, although an interpolar or lower polar posterior calyx is usually chosen. However, if PCN is to be followed by ureteral stent insertion an interpolar calyceal entry is preferable, so that the vector of pushing forces can be directed toward the ureteropelvic junction. Access through the upper pole calyces may be required in patients with stones, in which case an intercostal tract may be needed. Once the puncture site is chosen, local anaesthesia is liberally administered into the skin site and throughout the proposed track to the level of the renal fascia. In general practice urine sample of patients with UTI are not routinely tested and susceptibility testing is recommended only in cases of treatment failure or suspected complications. Consequently, in the absence of more accurate local antimicrobial susceptibility pattern, the results of this study could serve as 
a valuable source for antibacterial drugs prescription in empirical treatment of UTI.

\begin{tabular}{|c|c|}
\hline Mean Age (yrs.) & $15-55$ \\
\hline \multicolumn{2}{|l|}{ Gender } \\
\hline Male & 56 \\
\hline Female & 22 \\
\hline \multicolumn{2}{|l|}{ Side } \\
\hline Right & $44(56.4 \%)$ \\
\hline Left & $34(43.6 \%)$ \\
\hline \multicolumn{2}{|l|}{ Technique } \\
\hline Ultrasound & 78 \\
\hline \multicolumn{2}{|l|}{ Comorbidities } \\
\hline Urinary Tract Obstruction & $43(55 \%)$ \\
\hline Malignancy & $20(25 \%)$ \\
\hline Pregnancy & $4(5 \%)$ \\
\hline Other Causes & $11(15 \%)$ \\
\hline
\end{tabular}

\begin{tabular}{|c|c|c|c|}
\hline & $\begin{array}{c}\text { PCN Culture } \\
\text { +ve (n=76) }\end{array}$ & $\begin{array}{c}\text { Urethral Urine } \\
\text { Culture +ve (n=71) }\end{array}$ & P 0.09 \\
\hline Escherichia coli & $48(63 \%)$ & $44(62.5 \%)$ & \\
\hline Klebsiella spp. & $11(15 \%)$ & $10(15 \%)$ & \\
\hline $\begin{array}{c}\text { Pseudomonas } \\
\text { spp. }\end{array}$ & $8(9 \%)$ & $7(9 \%)$ & \\
\hline $\begin{array}{c}\text { Enterococcus } \\
\text { spp. }\end{array}$ & $3(4 \%)$ & $2(4 \%)$ & \\
\hline $\begin{array}{c}\text { Staphylococcus } \\
\text { spp. }\end{array}$ & $3(4 \%)$ & $2(4 \%)$ & \\
\hline $\begin{array}{c}\text { Acinetobacter } \\
\text { spp. }\end{array}$ & $1(.17 \%)$ & $1(.20 \%)$ & \\
\hline
\end{tabular}

Table 2. Organism Growth Culture Positivity Rates in Urine Samples obtained from PCN Tubes and Urethral Catheters

\section{CONCLUSION}

PCN is an important treatment for the management of pyonephrosis. Although, there was no statistically significant differences between groups, number of positive PCN urine cultures was higher when compared with urethral urine cultures. PCN cultures are associated with minor morbidity, provide therapeutic benefit and its use is recommended as a guiding tool for the re-treatment of pyonephrosis after failed medical therapy.

\section{REFERENCES}

[1] Neal DE. Complicated urinary tract infections. Urol Clin North Am 2008;35(1):13-22.

[2] Mazzulli T. Diagnosis and management of simple and complicated urinary tract infections (UTIs). Can J Urol 2012;19(1):42-8.

[3] Cox CE, Hinman F. Experiments with induced bacteriuria, vesical emptying and bacterial growth on the mechanism of bladder defense to infection. J Urol 1961;86:739-48.
[4] Ramchandani P, Cardella JF, Grassi CJ, et al. Quality improvement guidelines for percutaneous nephrostomy. J Vasc Interv Radiol. Campbell Walsh urology, Elsevier 2016.

[5] Larsen EH, Gasser TC, Madsen PO. Antimicrobial prophylaxis in urologic surgery. Urol Clin North Am 1986;13(4):591-604.

[6] Hugosson J, Grenabo L, Hedelin H, et al. Bacteriology of upper urinary tract stones. J Urol 1990;143(5):965-8

[7] McCartney AC, Clark J, Lewi HJ. Bacteriological study of renal calculi. Eur J Clin Microbiol 1985;4(6):553-5.

[8] Preminger GM, Assimos DG, Lingeman JE, et al. Chapter 1: AUA guideline on management of staghorn calculi: diagnosis and treatment recommendations. J Urol 2005;173(6):1991-2000.

[9] Bruce RR, Griffith DP. Retrospective follow up of patients with struvite calculi. In: Urolithiasis clinical and basic research. Smith LH, Robertson WGL, Finlayson B. (eds). New York: plenum press 1981:P 191.

[10] Charton M, Vallancien G, Veillon B, et al. Urinary tract infection in percutaneous surgery for renal calculi. J Urol 1986;135(1):15-7.

[11] Mariappan P, Smith G, Bariol SV, et al. Stone and pelvic urine culture and sensitivity are better than bladder urine as predictors of urosepsis following percutaneous nephrolithotomy: a prospective clinical study. J Urol 2005;173(5):1610-4.

[12] Sancaktutar AA, Bozkurt Y, Tüfek A, et al. Radiationfree percutaneous nephrostomy performed on neonates, infants, and preschool-age children. J Pediatr Urol 2013;9(4):464-71.

[13] Lewi HJ, White A, Hutchinson AG, et al. The bacteriology of the urine and renal calculi. Urol Res 1984;12(2):107-9.

[14] Fowler JE. Bacteriology of branched renal calculi and accompanying urinary tract infection. J Urol 1984;131(2):213-5.

[15] Gnessin E, Mandeville JA, Handa SE, et al. Changing composition of renal calculi in patients with musculoskeletal anomalies. J Endourol 2011;25(9):1519-23.

[16] Viprakasit DP, Sawyer MD, Herrell SD, et al. Changing composition of staghorn calculi. J Urol 2011;186(6):2285-90.

[17] Mandel N, Mandel I, Fryjoff K, et al. Conversion of calcium oxalate to calcium phosphate with recurrent stone episodes. J Urol 2003;169(6):2026-9.

[18] Stapleton AE. Urinary tract infection pathogenesis: host factors. Infect Dis Clin North Am 2014;28(1): 149-59. 